\section{Tornar-se obeso na adolescência pode trazer consequências à saúde mental?}

\author{
Can teenage obesity affect mental health?
}

\section{¿Convertirse en un obeso durante la adolescencia puede traer consecuencias para la salud mental?}

\author{
Maria Cecília Formoso Assunção 1 \\ Ludmila Correa Muniz 1 \\ Antônio Augusto Schäfer 1 \\ Fernanda de Oliveira Meller 1 \\ Juliana Pires Carús 1 \\ Lenice de Castro Muniz de Quadros 1 \\ Lídice Rodrigues Domingues 1 \\ Vera Lúcia Schmidt da Silva ${ }^{1}$ \\ Helen Gonçalves 1 \\ Pedro Curi Hallal 1 \\ Ana Maria Baptista Menezes 1
}

\author{
${ }_{1}$ Programa de Pós-graduação \\ em Epidemiologia, \\ Universidade Federal de \\ Pelotas, Pelotas, Brasil. \\ Correspondência \\ M. C. F. Assunção \\ Programa de Pós-graduação \\ em Epidemiologia, \\ Universidade Federal de \\ Pelotas. \\ Rua Marechal Deodoro 1.160, \\ 3o andar, Pelotas, RS 96020 - \\ 220, Brasil. \\ cecilia.epi@gmail.com
}

\begin{abstract}
This study evaluated the association between obesity and emotional and behavioral difficulties in adolescents. We studied 4,325 individuals 11 to 15 years of age who were members of the 1993 birth cohort in Pelotas, Rio Grande do Sul State, Brazil. Information on body mass index (BMI), maternal assessment of the adolescents' emotional and behavioral health (Strengths and Difficulties Questionnaire - SDQ), and sociodemographic and behavioral characteristics were used. Gender-stratified analyses were conducted with simple and multivariate linear regression. In the adjusted analysis, obesity only correlated with total SDQ scores in boys. Among the latter, teenage obesity was associated with higher scores on the subscale of relational problems with peers. Given current knowledge on the future implications of obesity and mental health and in dealing with adolescents, studies on gender differences in adolescence may contribute to understanding such associations.
\end{abstract}

Obesity; Adolescent Behavior; Mental Health

\section{Resumo}

O estudo avaliou a associação entre trajetória de obesidade e dificuldades emocionais e comportamentais em adolescentes. Foram estudados 4.325 jovens, aos 11 e 15 anos de idade, pertencentes à coorte de nascimentos de 1993 de Pelotas, Rio Grande do Sul, Brasil. Informações sobre indice de massa corporal (IMC), avaliação materna da saúde emocional e comportamental do adolescente (Strengths and Difficulties Questionnaire - SDQ) e características sociodemográficas e comportamentais foram utilizadas. As análises, estratificadas por sexo, foram conduzidas por meio de regressão linear simples $e$ múltipla. A trajetória de obesidade mostrou associação com o escore total do SDQ, nas análises ajustadas, apenas entre os meninos. Entre esses, tornar-se obeso no período esteve relacionado com maior escore na subescala de problemas de relacionamento com os colegas. Diante do conhecimento atual sobre as implicações futuras da obesidade na saúde mental e em se tratando de adolescentes, sugere-se que estudos que avaliem as diferenças de gênero na adolescência possam contribuir para o entendimento da associação encontrada.

Obesidade; Comportamento do Adolescente; Saúde Mental 


\section{Introdução}

As consequências, em curto e longo prazo, do sobrepeso e da obesidade sobre a saúde física são conhecidas 1 . No entanto, ainda existem controvérsias acerca do impacto da obesidade sobre a saúde mental dos indivíduos 2,3. Alguns estudos sugerem que a obesidade pode levar a problemas de saúde mental 4 , enquanto outros mostram que adolescentes que apresentam problemas de saúde mental são mais propensos à obesidade 5. Para estabelecer essa relação, são necessários estudos prospectivos, os quais ainda são escassos 3,6.

O interesse por estudos que analisam a relação entre obesidade e problemas de saúde mental tem sido crescente. Esses tentam explicar as relações causais entre obesidade e transtornos ou problemas mentais ao longo da vida, avaliando fatores sociais - como apoio familiar reduzido, sofrer estigma e/ou bullying; fatores comportamentais - fazer dieta e perder/ganhar peso; fatores biológicos - ter doenças crônicas e usar medicamentos e fatores psicológicos - autopercepção de saúde como ruim e insatisfação corporal 7,8.

Embora alguns estudos evidenciem que alterações comportamentais no decorrer da vida podem ser atribuídas à obesidade $4,7,8$, essa relação necessita de análises mais aprofundadas, considerando o efeito temporal e os elementos que possam influenciar o desenvolvimento da relação entre ambas.

Diante disso e da escassa literatura sobre esse tema, principalmente em adolescentes, o presente estudo objetivou avaliar um aspecto desse mecanismo, a associação entre trajetória de obesidade e dificuldades emocionais e comportamentais em adolescentes pertencentes a uma coorte de nascimentos da cidade de Pelotas, Rio Grande do Sul, Brasil. Também foi objetivo deste estudo identificar a existência de diferenças entre os sexos.

\section{Métodos}

Para o presente estudo, foram utilizados dados da coorte de nascimentos do ano de 1993, da cidade de Pelotas, referentes aos acompanhamentos realizados em 2004-2005 e em 2008, quando os participantes tinham 11-12 anos e 14-15 anos de idade, respectivamente. Em 2004-2005, dentre os 5.249 indivíduos da coorte original, foram localizados 4.452 adolescentes, atingindo uma taxa de resposta de $87,5 \%$ na qual foram considerados os óbitos ocorridos ao longo do período. Aos 15 anos, 4.325 jovens ( $85,7 \%$ da coorte original) foram entrevistados. Informações metodo- lógicas detalhadas sobre o estudo encontram-se em outra publicação ${ }^{9}$.

Para a avaliação da presença de sintomas que detectem problemas de saúde mental aos 15 anos de idade (desfechos do estudo), utilizou-se a versão do Strengths and Difficulties Questionnaire (SDQ) 10, aplicada às mães dos adolescentes, que permite mensurar características comportamentais e emocionais dos mesmos. O instrumento de rastreamento, já validado para uso em crianças e adolescentes brasileiros 11, contém 25 questões que englobam cinco subescalas (comportamento pró-social, hiperatividade/déficit de atenção, sintomas emocionais, problemas de conduta e de relacionamento com colegas). As opções de respostas são: falso, mais ou menos verdadeiro ou verdadeiro, e cada item recebe pontuação específica (variando de zero a dois pontos, atingindo até dez pontos por subescala se todos os itens forem respondidos). Exceto para comportamento pró-social, nas demais subescalas, quanto maior a pontuação, maior é o número de sintomas ${ }^{10}$. Para construir o escore total, são somadas quatro subescalas: déficit de atenção/hiperatividade, problemas emocionais, de conduta e de relacionamento com colegas. Os escores totais e de cada subescala do SDQ foram analisados de maneira contínua.

A avaliação do estado nutricional dos adolescentes, aos 11 e 15 anos de idade, foi realizada por meio do índice de massa corporal (IMC) para a idade em escore-z, segundo recomendação da Organização Mundial da Saúde para crianças e adolescentes de 5-19 anos 12. Foram classificados como obesos os adolescentes que apresentaram IMC para a idade maior que +2 escore- $z$. Os adolescentes foram pesados em balança eletrônica da marca Tanita (São Paulo, Brasil), com capacidade máxima de $150 \mathrm{~kg}$ e precisão de $100 \mathrm{~g}$. E a estatura foi medida por meio de um estadiômetro de alumínio, com capacidade de $2 \mathrm{~m}$ e precisão de $1 \mathrm{~mm}$. As medidas antropométricas foram coletadas por equipe treinada e padronizada.

Com base no estado nutricional dos jovens em ambos os períodos, foi construída a variável de exposição principal: "trajetória de obesidade dos 11 aos 15 anos”, ou seja, a mudança de categoria de IMC que o adolescente sofreu entre ambos os períodos avaliados. Essa variável foi gerada a partir da combinação da presença de obesidade aos 11 e/ou aos 15 anos de idade e categorizada em: "não obeso aos 11 e aos 15 anos", "não obeso aos 11 e obeso aos 15 anos", "obeso aos 11 e não obeso aos 15 anos" e "obeso aos 11 e aos 15 anos".

Para controle de confusão nas análises, foram utilizadas variáveis referentes ao acompanhamento dos 11 anos, as quais foram operacio- 
nalizadas da seguinte maneira: sexo (masculino ou feminino), cor da pele autorreferida (branca, preta/negra, outra), índice de bens (em quintis), escolaridade materna em anos completos de estudo $(0-4 ; 5-8 ; 9-11 ; 12$ ou mais), realização de regime para emagrecer no último ano (sim ou não), uso de remédio para emagrecer no último ano (sim ou não), autopercepção da imagem corporal (gostaria de ter uma silhueta menor, satisfeito com sua imagem corporal ou gostaria de ter uma silhueta maior), escore do SDQ total e das subescalas aos 11 anos de idade e escore do Self Report Questionnaire (SRQ) materno. O SRQ é um instrumento screening para transtornos mentais comuns, como ansiedade, depressão e sintomas somatoformes. O teste possui vinte questões com duas possibilidades de resposta ( $\operatorname{sim} /$ não), e o ponto de corte utilizado neste estudo para SRQ positivo materno foi maior que oito pontos, em acordo com a validação brasileira 13 .

A variável autopercepção da imagem corporal foi criada comparando-se a imagem corporal desejada com a imagem corporal percebida pelo adolescente, mensurada por meio da escala de silhuetas de Stunkard et al. 14. Essa escala contém nove figuras do sexo masculino e feminino, que variam da menor para a maior silhueta, sendo apresentadas de forma desordenada para os jovens. Inicialmente, os adolescentes escolhiam a figura com a qual acreditavam se parecer mais e, a seguir, elegiam a silhueta que desejavam ter. Se a resposta das duas questões fosse referente à mesma silhueta, considerava-se o adolescente como satisfeito com sua imagem corporal. Caso contrário, o adolescente era considerado insatisfeito com a própria imagem corporal, independente se o seu desejo fosse ter uma silhueta maior ou menor do que a autopercebida 14 .

As análises estatísticas foram realizadas utilizando-se o software Stata 12.0 (Stata Corp., College Station, Estados Unidos). Foram realizados a análise descritiva da amostra e, posteriormente, o teste t não pareado para verificar a diferença entre as médias do escore total e das subescalas do SDQ entre os sexos. Modelos de regressão linear simples e de regressão linear múltipla foram propostos para verificar a associação da trajetória de obesidade e sintomas para dificuldades emocionais e comportamentais entre os adolescentes. As análises foram estratificadas por sexo. Adotou-se um nível de 5\% de significância.

Um Termo de Consentimento Livre e Esclarecido foi assinado pelas mães e/ou responsáveis pelos adolescentes, em ambos os acompanhamentos, e o projeto foi aprovado pelo Comitê de Ética e Pesquisa da Faculdade de Medicina da Universidade Federal de Pelotas (ofício no 158/07).

\section{Resultados}

Dos 4.325 adolescentes incluídos na análise (aos 11 anos), pouco mais da metade $(51,1 \%)$ era do sexo feminino, e dois terços tinham cor da pele branca. Cerca de um quarto das mães possuía escolaridade inferior a quatro anos de estudo. Aproximadamente, 9\% dos jovens referiram ter realizado regime com objetivo de perder peso no último ano, menos de $1 \%$ informaram ter feito uso de remédio para emagrecer nos últimos 12 meses, e 57,5\% se mostraram insatisfeitos com sua imagem corporal, ou seja, desejavam, aos 11 anos, ter uma silhueta maior ou menor (Tabela 1).

A Tabela 2 mostra que a média do escore total do SDQ, para toda a amostra, foi de 12,10 pontos (IC95\%: 11,89; 12,31), sendo maior entre as meninas $(12,33$; IC95\%: 12,03; 12,63) quando comparadas aos meninos (11,86; IC95\%: 11,56; 12,16). Quanto às subescalas que compõem o SDQ, apenas as médias para comportamento pró-social não diferiram entre os sexos (valor de $\mathrm{p}=0,529$ ). Para as demais subescalas, somente a média do escore para hiperatividade/déficit de atenção foi maior entre os meninos (valor de $\mathrm{p}<0,001$ ), sendo as demais maiores entre as meninas.

A análise bruta e ajustada da associação entre o escore total do SDQ e a trajetória de obesidade, geral e estratificada por sexo, está apresentada na Tabela 3. Na amostra total, a associação perdeu a significância após análise ajustada (valor de $\mathrm{p}=0,065$ ), todavia, os adolescentes que se tornaram obesos apresentaram um escore total de 1,17 ponto maior do que aqueles não obesos nos dois períodos (coeficiente ajustado: 1,17; IC95\%: 0,$07 ; 2,27)$. Entre as meninas, a associação permaneceu não significativa. Entre os meninos, aqueles que se tornaram obesos entre os 11 e os 15 anos de idade apresentaram um escore total do SDQ de 1,71 ponto maior do que aqueles não obesos em ambos os períodos (coeficiente ajustado: 1,71; IC95\%: 0,10; 3,33).

A Tabela 4 apresenta a análise ajustada da associação entre as subescalas do SDQ e a trajetória de obesidade conforme o sexo. Evidenciou-se que apenas os problemas de relacionamento entre os meninos mostraram-se associados à trajetória de obesidade, ou seja, aqueles que se tornaram obesos aos 15 anos apresentaram maior escore de problemas de relacionamento comparados aos não obesos aos 11 e 15 anos de idade. O mesmo foi observado para os meninos que deixaram de ser obesos, embora em menor magnitude. 
Características demográficas, socioeconômicas e comportamentais de adolescentes aos 11 anos da coorte de nascimentos de 1993. Pelotas, Rio Grande do Sul, Brasil, 2004 ( $N=4.325$ *).

\begin{tabular}{|c|c|c|}
\hline Variáveis & n & $\%$ \\
\hline \multicolumn{3}{|l|}{ Sexo } \\
\hline Masculino & 2.113 & 48,9 \\
\hline Feminino & 2.212 & 51,1 \\
\hline \multicolumn{3}{|l|}{ Cor da pele } \\
\hline Branca & 2.810 & 66,7 \\
\hline Preta/Negra & 528 & 12,5 \\
\hline Outra & 879 & 20,8 \\
\hline \multicolumn{3}{|l|}{ Índice de bens (quintis) } \\
\hline 1으 (menor) & 810 & 19,8 \\
\hline 2으 & 808 & 19,7 \\
\hline 3으 & 831 & 20,2 \\
\hline 4으 & 826 & 20,2 \\
\hline 5으 (maior) & 823 & 20,1 \\
\hline \multicolumn{3}{|l|}{ Escolaridade materna (anos) } \\
\hline $0-4$ & 1.070 & 25,4 \\
\hline $5-8$ & 1.829 & 43,4 \\
\hline $9-11$ & 911 & 21,6 \\
\hline 12 ou mais & 407 & 9,6 \\
\hline \multicolumn{3}{|l|}{ Fez regime para emagrecer no último ano } \\
\hline Não & 3.875 & 91,4 \\
\hline Sim & 364 & 8,6 \\
\hline \multicolumn{3}{|c|}{ Usou remédio para emagrecer no último ano } \\
\hline Não & 4.223 & 99,6 \\
\hline Sim & 16 & 0,38 \\
\hline \multicolumn{3}{|l|}{ Autopercepção da imagem corporal } \\
\hline Gostaria de ter uma silhueta menor & 900 & 21,3 \\
\hline Satisfeito com sua imagem corporal & 1.799 & 42,5 \\
\hline \multirow[t]{2}{*}{ Gostaria de ter uma silhueta maior } & 1.528 & 36,2 \\
\hline & Média & IC95\% \\
\hline Escore SRQ materno & 5,70 & $5,56-5,84$ \\
\hline
\end{tabular}

IC95\%: intervalo de 95\% de confiança; SRQ: Self Report Questionnaire.

* Considerados os adolescentes que também foram entrevistados aos 15 anos;

Nota: percentual máximo de observações desconhecidas: $5,2 \%(n=227)$ para a variável índice de bens

\section{Discussão}

O presente estudo evidenciou associação entre a trajetória de obesidade e dificuldades emocionais e comportamentais, avaliadas pelo SDQ em algumas subescalas. Na de relacionamento com colegas, a associação estudada foi evidenciada na amostra total e nos meninos. Portanto, aqueles meninos que se tornaram obesos aos 15 anos apresentaram maiores escores desse problema quando comparados às demais categorias e às meninas. Tal associação sugere que os que se tornaram obesos aos 15 anos ("não obeso aos
11 e obeso aos 15 anos") estão mais propensos a demonstrar insatisfações nas suas relações sociais e afetivas ou por meio delas responderem às adversidades, mostrando que a obesidade pode apresentar repercussões distintas entre os sexos e faixa etária.

Diante do conhecimento atual sobre as implicações futuras tanto da obesidade quanto da saúde mental e em se tratando de adolescentes, sugere-se que estudos que avaliem as diferenças de gênero nesse período da adolescência possam contribuir para o entendimento da associação encontrada. Outro trabalho com pertencentes 
Média do escore total e subescalas do Strengths and Difficulties Questionnaire (SDQ) em adolescentes de 15 anos da coorte de nascimentos de 1993. Pelotas, Rio Grande do Sul, $2008(N=4.325)$ *

\begin{tabular}{|c|c|c|c|c|}
\hline SDQ total e subescalas & $\begin{array}{c}\text { Geral } \\
\text { Média (IC95\%) }\end{array}$ & $\begin{array}{c}\text { Meninos } \\
\text { Média (IC95\%) }\end{array}$ & $\begin{array}{c}\text { Meninas } \\
\text { Média (IC95\%) }\end{array}$ & $\begin{array}{c}\text { Valor de } \\
p^{\star \star}\end{array}$ \\
\hline Escore total & $12,10(11,89 ; 12,31)$ & $11,86(11,56 ; 12,16)$ & $12,33(12,03 ; 12,63)$ & 0,030 \\
\hline Sintomas emocionais & $3,94(3,86 ; 4,02)$ & $3,47(3,36 ; 3,58)$ & $4,39(4,28 ; 4,51)$ & $<0,0001$ \\
\hline Problemas de conduta & $2,28(2,21 ; 2,35)$ & $2,17(2,08 ; 2,27)$ & $2,38(2,29 ; 2,48)$ & 0,002 \\
\hline Hiperatividade/Déficit de atenção & $3,80(3,71 ; 3,89)$ & $4,21(4,08 ; 4,35)$ & $3,40(3,28 ; 3,52)$ & $<0,0001$ \\
\hline Problemas de relacionamento & $2,08(2,02 ; 2,14)$ & $2,00(1,92 ; 2,08)$ & $2,16(2,07 ; 2,24)$ & 0,010 \\
\hline Comportamento pró-social & $8,56(8,51 ; 8,62)$ & $8,55(8,47 ; 8,63)$ & $8,58(8,50 ; 8,66)$ & 0,529 \\
\hline
\end{tabular}

IC95\%: intervalo de 95\% de confiança.

* Percentual máximo de observações desconhecidas: 0,3\% $(n=12)$ para as variáveis escore total e problemas de

relacionamento;

** Diferença entre meninos e meninas estimada pelo Teste $t$

Tabela 3

Análise bruta e ajustada das trajetórias de obesidade e do escore total do Strengths and Difficulties Questionnaire (SDQ), para o total da amostra e estratificada por sexo, em adolescentes de 15 anos da coorte de nascimentos de 1993. Pelotas, Rio Grande do Sul, Brasil, 2008 (N = 4.325).

\begin{tabular}{|c|c|c|c|c|c|c|}
\hline \multirow{3}{*}{$\begin{array}{l}\text { Trajetórias de obesidade } \\
\text { (11-15 anos) }\end{array}$} & \multicolumn{2}{|c|}{ Geral } & \multicolumn{2}{|c|}{ Meninos } & \multicolumn{2}{|c|}{ Meninas } \\
\hline & Bruta & Ajustada * & Bruta & Ajustada * & Bruta & Ajustada * \\
\hline & $\beta$ (IC95\%) & $\beta$ (IC95\%) & $\beta$ (IC95\%) & B (IC95\%) & 及 (IC95\%) & $\beta$ (IC95\%) \\
\hline & $p=0,024 * \star$ & $p=0,065 \star \star$ & $p=0,092^{* \star}$ & $p=0,032 * \star$ & $p=0,073 \star \star \star$ & $p=0,734 * \star$ \\
\hline Não obeso - Não obeso & Referência & Referência & Referência & Referência & Referência & Referência \\
\hline Obeso - Não obeso & $-0,32(-1,39 ; 0,74)$ & $0,57(-0,30 ; 1,44)$ & $0,30(-1,06 ; 1,66)$ & $1,06(-0,07 ; 2,18)$ & $-1,14(-2,84 ; 0,57)$ & $-0,20(-1,59 ; 1,19)$ \\
\hline Obeso - Obeso & $0,53(-0,85 ; 0,96)$ & $-0,35(-1,14 ; 0,43)$ & $-0,37(-1,51 ; 0,77)$ & $-0,36(-1,37 ; 0,65)$ & $1,00(-0,50 ; 2,50)$ & $-0,27(-1,55 ; 1,00)$ \\
\hline
\end{tabular}

SRQ: Self Report Questionnaire.

* Ajustado para cor da pele, índice de bens, escolaridade materna, regime no último ano, uso de remédio para emagrecer no último ano, autopercepção da imagem corporal, escore SRQ materno e escore SDQ aos 11 anos.

** Valor de p estimado pelo Teste de Wald.

a essa mesma coorte demonstrou que, aos 18 anos, os adolescentes homens enfatizavam suas preocupações com a aparência mais do que as meninas, deixando claro que as exigências para estar em padrão não gordo foram estendidas também a eles. Esse aspecto, no entanto, pode estar ocorrendo mais precocemente na adolescência, quando, aos 15 anos, as cobranças para o namoro e outros envolvimentos e o posicionamento social passam pelo "visual". Esse tipo de cobrança, quando internalizada, pode gerar mudanças e reações no comportamento. Prevenções na área de saúde mental e nutrição devem considerar que a obesidade também traz consequências psicológicas aos meninos.

O presente trabalho possui uma amostra representativa e alta taxa de seguimento, suas informações - coletadas de forma prospectiva minimizam o viés de memória e permitem identificar a temporalidade das associações.

O impacto da obesidade sobre a saúde mental e vice-versa já está comprovado 2,3,4,5. Novas investigações sobre os mecanismos e fatores que estão influenciando nas relações ainda são necessárias. Outros estudos longitudinais também têm evidenciado associação entre obesidade e 
Tabela 4

Análise ajustada * das trajetórias de obesidade e das subescalas do Strengths and Difficulties Questionnaire (SDQ), estratificada por sexo, em adolescentes de 15 anos da coorte de nascimentos de 1993. Pelotas, Rio Grande do Sul, Brasil, 2008 ( $N=4.325)$.

\begin{tabular}{|c|c|c|c|c|c|}
\hline $\begin{array}{l}\text { Trajetórias de obesidade } \\
(11-15 \text { anos)/Subescalas }\end{array}$ & $\begin{array}{l}\text { Sintomas } \\
\text { emocionais }\end{array}$ & $\begin{array}{l}\text { Problemas de } \\
\text { conduta }\end{array}$ & $\begin{array}{l}\text { Hiperatividade/ } \\
\text { Déficit de atenção }\end{array}$ & $\begin{array}{l}\text { Problemas de } \\
\text { relacionamento }\end{array}$ & $\begin{array}{c}\text { Comportamento } \\
\text { pró-social }\end{array}$ \\
\hline SDQ & B (IC95\%) & B (IC95\%) & $\beta$ (IC95\%) & 及 (IC95\%) & $\beta$ (IC95\%) \\
\hline Meninos & $p=0,294 * \star$ & $p=0,099 \star \star$ & $p=0,389 * *$ & $p=0,005 * \star$ & $\mathrm{p}=0,921 \star \star \star$ \\
\hline Não obeso - Não obeso & Referência & Referência & Referência & Referência & Referência \\
\hline Não obeso - Obeso & $0,55(-0,10 ; 1,19)$ & $0,09(-0,44 ; 0,63)$ & $0,42(-0,33 ; 1,17)$ & $0,78(0,27 ; 1,28)$ & $0,14(-0,37 ; 0,64)$ \\
\hline Obeso - Não obeso & $0,10(-0,35 ; 0,55)$ & $0,25(-0,13 ; 0,63)$ & $0,37(-0,16 ; 0,89)$ & $0,39(0,04 ; 0,74)$ & $0,04(-0,31 ; 0,39)$ \\
\hline Obeso - Obeso & $-0,14(-0,54 ; 0,26)$ & $-0,31(-0,64 ; 0,03)$ & $-0,01(-0,47 ; 0,47)$ & $0,16(-0,15 ; 0,48)$ & $0,08(-0,24 ; 0,39)$ \\
\hline Meninas & $\mathrm{p}=0,674^{\star \star}$ & $p=0,534^{\star \star}$ & $\mathrm{p}=0,591 \star \star$ & $p=0,364^{\star \star}$ & $p=0,903 * \star$ \\
\hline Não obeso - Não obeso & Referência & Referência & Referência & Referência & Referência \\
\hline Não obeso - Obeso & $0,25(-0,37 ; 0,86)$ & $0,27(-0,26 ; 0,80)$ & $-0,16(-0,81 ; 0,49)$ & $0,39(-0,10 ; 0,89)$ & $0,12(-0,35 ; 0,59)$ \\
\hline Obeso - Não obeso & $0,21(-0,35 ; 0,78)$ & $-0,06(-0,55 ; 0,43)$ & $-0,36(-0,95 ; 0,23)$ & $-0,02(-0,48 ; 0,43)$ & $0,13(-0,30 ; 0,56)$ \\
\hline Obeso - Obeso & $-0,14(-0,66 ; 0,38)$ & $-0,23(-0,68 ; 0,22)$ & $0,11(-0,44 ; 0,66)$ & $0,20(-0,22 ; 0,61)$ & $0,04(-0,35 ; 0,44)$ \\
\hline
\end{tabular}

* Ajustado para cor da pele, índice de bens, escolaridade materna, regime no último ano, uso de remédio para emagrecer no último ano, autopercepção da imagem corporal, escore SRQ materno e subescalas do SDQ correspondente aos 11 anos.

** Valor de p estimado pelo Teste de Wald.

saúde mental 15,16,17,18. Swallen et al. ${ }^{17}$, por exemplo, observaram impacto negativo do sobrepeso e da obesidade na autoestima e no desenvolvimento social e escolar de adolescentes. Smith et al. 18 evidenciaram associação entre bullying e problemas de relacionamento em adolescentes, sendo que os meninos apresentaram maiores escores do que as meninas. Segundo Drukker et al. 6, existem indicadores de que algumas áreas da psicopatologia podem estar diferentemente associadas à obesidade.

Estudos transversais com crianças e adolescentes também evidenciaram associação entre obesidade e saúde mental 6,19. Na Espanha, adolescentes obesos apresentaram mais dificuldades emocionais e comportamentais ao utilizarem o SDQ 19; na Holanda, adolescentes com sobrepeso ou obesidade reportaram mais problemas de relacionamento ${ }^{6}$. Em contraposição, os resultados de um estudo longitudinal, realizado na Inglaterra, observou que sobrepeso e obesidade não estiveram associados à saúde psicológica de adolescentes 20 .

Cabe ressaltar novamente que, neste estudo, a aplicação do SDQ às mães dos adolescentes ocorreu nos dois acompanhamentos, ao passo que os jovens responderam aos 11 anos. Todavia, a versão dos pais possui sensibilidade de $62,1 \%$ para detectar qualquer distúrbio psiquiátrico e $73,5 \%$ de sensibilidade para detectar comportamento clinicamente significativo 21. Portanto, a subestimativa da prevalência de dificuldades emocionais e a superestimativa das comportamentais não são procedentes. Destaca-se que a aplicação desse instrumento aos adolescentes aos 15 anos não foi possível, especialmente, pelo fato de se tratar de um estudo com elevado número de entrevistados e uma grande quantidade de perguntas. Também não foram coletadas informações sobre a saúde mental do pai ou se o mesmo tinha algum problema mental/ de comportamento. A avaliação sobre fatores genéticos e de hereditariedade, os quais podem estar associados a problemas de relacionamento em crianças e adolescentes, será investigada clinicamente no futuro. No entanto, é importante considerar que, por não ser um instrumento diagnóstico, os resultados do SDQ indicam a presença de um conjunto de sintomas que podem apontar para o desenvolvimento de algum tipo de transtorno mental 22. 


\section{Resumen}

El estudio examinó la asociación entre la trayectoria de obesidad y las dificultades emocionales y de comportamiento en adolescentes. Se estudiaron 4.325 jóvenes, entre 11 y 15 años de edad, pertenecientes a la cohorte de nacimientos de 1993 en Pelotas, Brasil. Información acerca del índice de masa corporal (IMC), la evaluación materna de la salud emocional y comportamental de los adolescentes (Strengths and Difficulties Questionnaire - SDQ) y las características sociodemográficas y de comportamiento fueron utilizados. El análisis, estratificado por sexo, fue realizado a traves de la regresión lineal simple y múltiple. En el análisis ajustado, la trayectoria de la obesidad se há asociado com la puntuacion total del SDQ sólo entre los varones. Entre ellos, convertirse en obeso en el periodo se asoció con mayores puntuaciones en la subescala de problemas de relacionamiento con sus compañeros. Teniendo en cuenta los conocimientos actuales sobre las futuras consecuencias de la obesidad en la salud mental, y cuando se trata adolescentes, se sugiere que los estudios que evalúan las diferencias de género en la adolescencia puede contribuir a la comprensión de la asociación encontrada.

Obesidad; Conducta del Adolescente; Salud Mental

\section{Colaboradores}

Todos os autores participaram da concepção do estudo, revisaram versões preliminares do texto e das tabelas, fizeram sugestões de análises e aprovaram a versão final do manuscrito, submetida para publicação.

\section{Agradecimentos}

O estudo de coorte teve o apoio da Wellcome Trust e do CNPq. Nenhuma dessas organizações influenciou o desenho do estudo, a análise e interpretação de dados ou a decisão de submeter o manuscrito à publicação.

\section{Referências}

1. World Health Organization. Diet, nutrition and the prevention of chronic diseases. Geneva: World Health Organization; 2003.

2. McLaren L, Beck CA, Patten SB, Fick GH, Adair CE. The relationship between body mass index and mental health. A population-based study of the effects of the definition of mental health. Soc Psychiatry Psychiatr Epidemiol 2007; 43:63-71.

3. de Wit LM, van Straten A, van Herten M, Penninx BW, Cuijpers P. Depression and body mass index, a u-shaped association. BMC Public Health 2009; 9:14.

4. Scott KM, Bruffaerts R, Simon GE, Alonso J, Angermeyer M, de Girolamo G, et al. Obesity and mental disorders in the general population: results from the world mental health surveys. Int J Obes (Lond) 2008; 32:192-200.

5. Kiessling SG, McClanahan KK, Omar HA. Obesity, hypertension, and mental health evaluation in adolescents: a comprehensive approach. Int J Adolesc Med Health 2008; 20:5-15.
6. Drukker M, Wojciechowski F, Feron FJ, Mengelers R, Van Os J. A community study of psychosocial functioning and weight in young children and adolescents. Int J Pediatr Obes 2009; 4:91-7.

7. Hoek HW, van Harten PN, Hermans KM, Katzman MA, Matroos GE, Susser ES. The incidence of anorexia nervosa on Curacao. Am J Psychiatry 2005; 162:748-752.

8. Jansen W, Looij-Jansen PMV, Wilde EJ, Brug J. Feeling fat rather than being fat may be associated with psychological well-being in young Dutch adolescents. J Adolesc Health 2008; 42:128-36.

9. Victora CG, Hallal PC, Araujo CL, Menezes AMB, Wells JC, Barros FC. Cohort profile: the 1993 Pelotas (Brazil) birth cohort study. Int J Epidemiol 2008; 37:704-9.

10. Goodman R. The Strengths and Difficulties Questionnaire: a research note. J Child Psychol Psychiatry 1997; 38:581-6. 
11. Fleitlich B, Cortázar PG, Goodman R. Questionário de capacidades e dificuldades (SDQ). Infanto Rev Neuropsiquiatr Infanc Adolesc 2000; 8:44-50.

12. World Health Organization. WHO Growth reference data for 5-19 years. Geneva: World Health Organization; 2007.

13. Mari JJ, Williams P. A validity study of a psychiatric screening questionnaire (SRQ-20) in primary care in the city of São Paulo. Br J Psychiatry 1986; 148:23-6.

14. Stunkard AJ, Sorensen T, Schulsinger F. Use of Danish adoption register for the study of obesity and thinness. In: Kety S, editor. The genetics of neurological and psychiatric disorders. New York: Raven Press; 1983. p. 115-20.

15. Griffiths LJ, Dezateux C, Hill A. Is obesity associated with emotional and behavioral problems in children? Findings from the Millennium Cohort Study. Int J Pediatr Obes 2011; 6:423-32.

16. Sawyer MG, Harchak T, Wake M, Lynch J. Four-year prospective study of BMI and mental health problems in young children. Pediatrics 2011; 128:67784.

17. Swallen KC, Reither EN, Haas SA, Meier AM. Overweight, obesity, and health-related quality of life among adolescents: the national longitudinal study of adolescent health. Pediatrics 2005; 115:340-7.
18. Smith PK, Talamelli L, Cowie, H, Naylor P, Chauhan P. Profiles of non-victims, escaped victims, continuing victims and new victims of school bullying. Br J Educ Psychol 2004; 74:565-81.

19. Rodríguez-Hernández A, Cruz-Sánchez E, Feu S, Martínez-Santos R. Sedentarismo, obesidad y salud mental en la población española de 4 a 15 años de edad. Rev Esp Salud Pública 2011; 85:373-82.

20. Clark C, Haines MM, Head J, Klineberg E, Arephin M, Viner R, et al. Psychological symptoms and physical health and health behaviours in adolescents: a prospective 2-year study in East London. Addiction 2007; 102:126-35.

21. Tiffin PA, Arnott B, Moore HJ, Summerbell CD. Modelling the relationship between obesity and mental health in children and adolescents: findings from the Health Survey for England 2007. Child Adolesc Psychiatry Ment Health 2011; 5:31.

22. Goodman R, Meltzer H, Bailey V. The strengths and difficulties questionnaire: a pilot study on the validity of the self-report version. Eur Child Adoles Psy 1998; 7:125-30.

Recebido em 13/Set/2012

Versão final reapresentada em 04/Fev/2013

Aprovado em 20/Mar/2013 